\title{
GROSS ANATOMY OF LIVER, LUNG AND KIDNEY OF INDIAN COBRA (NAJA NAJA)
}

\author{
A. BANERJEE ${ }^{* 1}$ AND C. GHOSH ${ }^{2}$ \\ ${ }^{1}$ Block Animal Health Centre, Purbasthali-I, Purba Barddhaman, Pin-741 316, West Bengal, India \\ ${ }^{2}$ West Bengal University of Animal and Fishery Sciences, Kolkata-700 037, West Bengal, India
}

\begin{abstract}
Gross anatomical study of liver, lung and kidney of Indian cobra was conducted at Regional Laboratory, Jalpaiguri, West Bengal. The samples were collected from the accidental cases and preserved in $\mathbf{1 0 \%}$ formalin for further histological studies. Liver was elongated and tuber like structure was situated at the second quarter of the body. It was located in between the heart and stomach. Gall bladder was not associated with the liver like other domestic animals, located near the posterior tip of the liver. Spleen was a small spherical reddish organ located between the gall bladder and pancreas. Lung was elongated and the cranial part has a respiratory function where oxygen exchange is occurred. But the caudal part was avascular and consisted of many air sacs. Lung was located at the second and third quarters of the body. Both kidneys were lobulated, elongated and metanephric, and situated at the fourth quarter of the body. Right kidney was closer to the head than the left one.
\end{abstract}

Key word: Anatomy, Indian cobra, Kidney, Liver, Lungs

Indian cobra also known as spectacled cobra, Asian cobra or binocellate cobra is a species of the genus Naja found in India, Pakistan, Bangladesh, Srilanka, Nepal and Bhutan (Sapkota, 2019). It is a member of "Big four" species that inflict the most snake bites on humans in India. Indian cobra is revered in Indian mythology and culture and is often seen in the "Snake Charmer" (Khan, 2016). The literature on the gross anatomy of different organs of Indian cobra is very scanty. Therefore, considering the importance of this species of wildlife the present work was undertaken to elucidate the gross anatomical characteristics of the liver, lung and kidney.

A morbid specimen of a large Indian cobra was brought to the Regional Laboratory, Jalpaiguri, Animal Resources Development Department, Government of West Bengal. It was died in an accident in the local tea garden area of Jalpaiguri region. It was cleaned, washed and then fixed in $10 \%$ Neutral buffered formalin. After complete fixation the whole body was cut carefully to expose the liver, kidneys and lungs for anatomical study. Gross anatomical observations were recorded with respect to biometrical parameters, shape, external surfaces, color, location and relation with other internal organs. Biometrical parameters viz length, width were recorded by vernier caliper and weight by digital analytical balance.

Liver: The whole length of the Indian cobra was $170.18 \mathrm{~cm}$ and colour was dark glossy blackish brown dorsally and light brown ventrally. The liver was situated in the second quarter of the body filling the space in between the heart and stomach (Fig. 1). The liver was covered by a thick dense capsule (Fig. 2). The shape of the liver was

\footnotetext{
${ }^{*}$ Corresponding Author
} 
Indian Journal of Animal Health, June, 2020

Gross Anatomy of Liver, Lung and Kidney of Indian cobra



Fig. 1. Photograph showing the location of liver of Indian cobra in respect of other organ (H-Heart, L- Liver, DT- Digestive tube)



Fig. 2. Photograph showing gross anatomical structure of liver of Indian cobra showing elongated groove (G) in the parietal surface and capsule (C)



Fig. 3. Location of right lung of Indian cobra (dark congested area)

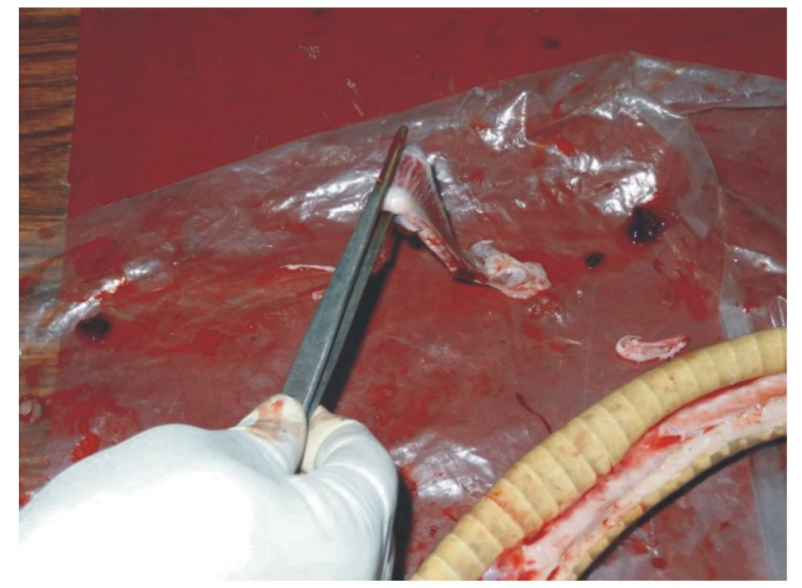

Fig. 4. Flat elongated right lung 
Indian Journal of Animal Health, June, 2020

Gross Anatomy of Liver, Lung and Kidney of Indian cobra

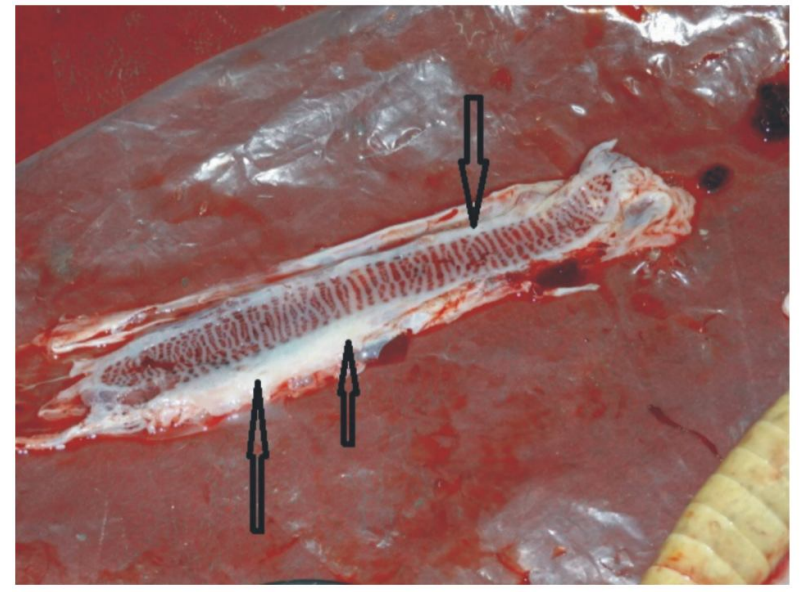

Fig. 5. Right lung of Indian cobra covered by whitish structure (Showing arrow mark)

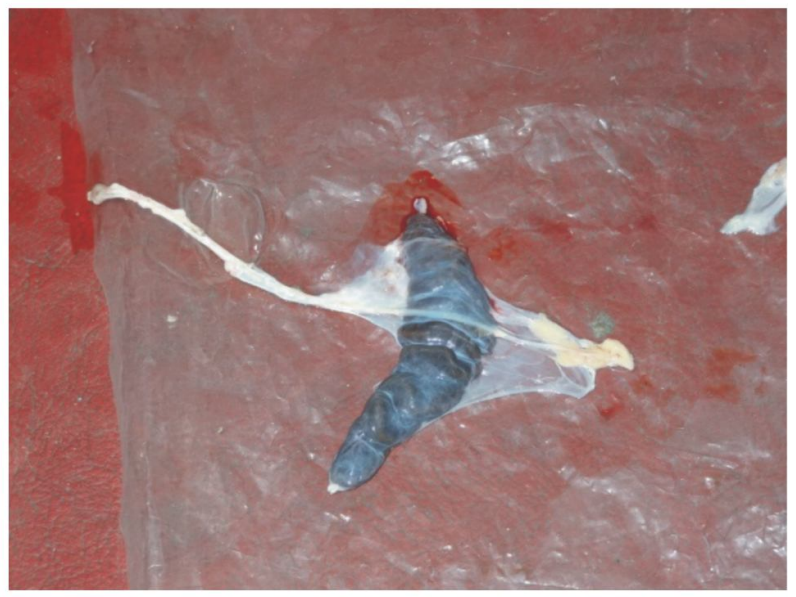

Fig. 6. Right kidney of Indian cobra

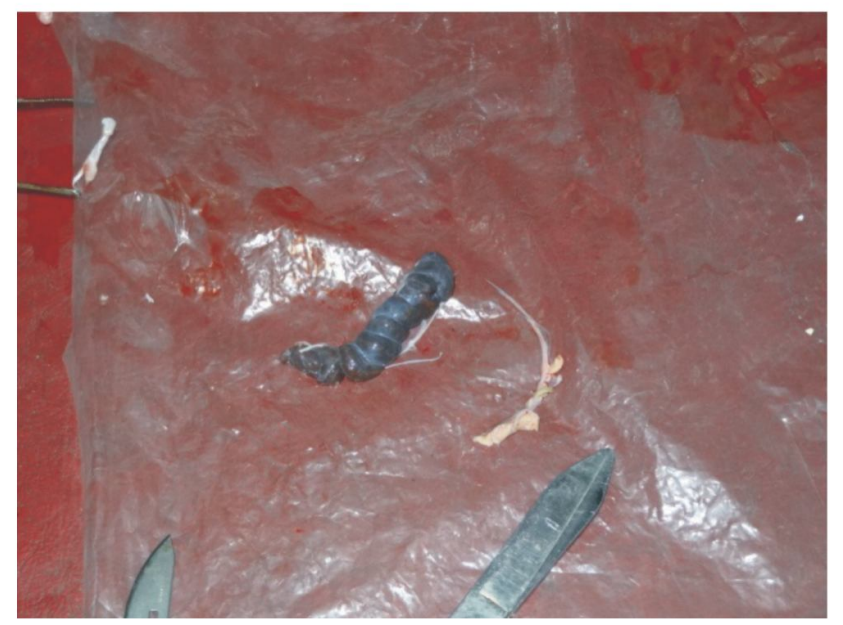

Fig. 7. Left kidney of Indian cobra 
elongated and tuber likes (Fig. 2). On macroscopical observation of the liver, it was revealed that there were two surfaces, two borders and two ends. These findings corroborated with the findings of Rajathi et al. (2015) in the liver of Cobra (Naja naja). The parietal and visceral surface was highly convex. In the middle of parietal surface, an elongated groove was present (Fig. 2). The length of matured liver was 17.3 $\mathrm{cm}$ and width was $1.24 \mathrm{~cm}$ in the middle portion. The weight was $71.36 \mathrm{gm}$. Two ends were prominent. Cranial end related to the large right lung and caudal end related to the duodenum, gall-bladder and partially to the stomach. The cranial and caudal borders were rounded.

Gall bladder was a well-developed muscular sac like structure adjacent to the duodenum and caudal to the liver. Multiple bile ducts came from the gall bladder and terminated into the duodenum. Gall bladder was not associated with the liver like other domestic animals. It was confined near the posterior tip of the liver. The spleen was small spherical reddish organ located between the gall bladder and pancreas.

Lung: Respiratory system of snake includes the trachea, bronchi, lungs and air sacs. Trachea of snake is popularly known as windpipe. The trachea started from the back of the oral cavity, and ended near the heart, where it branched into two bronchi-left and right. The left bronchus led to the left lung and right one for right lung. Lung

\section{REFERENCES}

Kings AS and McLelland J, 1975. Outline of Avian anatomy. Bailler Tindall, London, pp 67-69

Rajathi S, Kumaravel A and Muthukrishnan S, 2015. Morphological and morph metrical studies on the liver of Cobra (Naja naja). XXX Annual convention of Indian Association of Veterinary Anatomists and National Symposium on Recent advances in veterinary anatomy and their application in the field of animal health

Khan SM, 2016. The craft of snake charmar. The craft was extended from the second to third quarter of the body (Fig. 3).

Left lung was very small or completely rudimentary and non functioning. Similar findings were observed by Deka et al. (2017). The right lung was flat, elongated and functional (Fig. 4). Lung was covered by a whitish structure similar to pleura (Fig. 5). Similar findings were reported by Kings and McLelland (1975) in domestic fowl.

The cranial portion of the right lungs was vascular (with blood vessels) and may function in gaseous exchange, but the second half of the lung was an avascular (without blood vessels) air sac which extended into the tail region.

Kidney: The kidneys were elongated, lobulated in shape and triangular in cross section, located on either side of the large intestine (Fig. $6 \& 7$ ). The right kidney situated closer to the head than the left one. Both kidneys were covered by a thick capsule (Fig. $6 \& 7$ ). The ureters were hollow tubes which emptied directly in the cloacae (uorodeum). Weight of the right kidney was $26.3 \mathrm{gm}$ and the left kidney was $25.2 \mathrm{gm}$.

\section{ACKNOWLEDGEMENT}

Authors are extremely grateful to the Director of Animal Husbandry and Veterinary Services, Government of West Bengal and Deputy Director, ARD \& PO, Jalpaiguri for providing technical support to complete the study.

of snake charmers, pp 1-10, https:// www.academia.edu/

Deka A, Shrma K, Panchoni A, Kalita D and Ahmed J, 2017. Macro anatomical study on the heart, lung, trachea, kidney and liver of common rat snake (Ptyas mucosa). Therigenology Insight, $7(1): 1-4$

Sapkota S and Gurung DB, 2019. Important snake of Bhutan. Poster published in February 2019, doi: 10.13140/RG.2.2.23733.32481

Received-13.03.2020, Accepted-20.05. 2020, Published-01.06.2020 